\title{
Spotting high-z molecular absorbers using neutral carbon
}

\section{Results from a complete spectroscopic survey with the VLT^}

\author{
P. Noterdaeme ${ }^{1}$, C. Ledoux ${ }^{2}$, S. Zou ${ }^{1}$, P. Petitjean ${ }^{1}$, R. Srianand ${ }^{3}$, S. Balashev ${ }^{4}$, and S. López ${ }^{5}$ \\ ${ }^{1}$ Institut d'Astrophysique de Paris, CNRS-UPMC, UMR7095, 98bis boulevard Arago, 75014 Paris, France \\ e-mail: noterdaeme@iap.fr \\ ${ }^{2}$ European Southern Observatory, Alonso de Córdova 3107, Vitacura, Casilla 19001, Santiago 19, Chile \\ ${ }^{3}$ Inter-University Centre for Astronomy and Astrophysics, Post Bag 4, Ganeshkhind, 411007 Pune, India \\ ${ }^{4}$ Ioffe Institute, Polyteknicheskaya 26, 194021 Saint-Petersburg, Russia \\ ${ }^{5}$ Departamento de Astronomía, Universidad de Chile, Casilla 36-D, Santiago, Chile
}

Received 9 November 2017 / Accepted 22 January 2018

\begin{abstract}
While molecular quasar absorption systems provide unique probes of the physical and chemical properties of the gas as well as original constraints on fundamental physics and cosmology, their detection remains challenging. Here we present the results from a complete survey for molecular gas in thirty-nine absorption systems selected solely upon the detection of neutral carbon lines in Sloan Digital Sky Survey (SDSS) spectra, without any prior knowledge of the atomic or molecular gas content. $\mathrm{H}_{2}$ is found in all twelve systems (including seven new detections) where the corresponding lines are covered by the instrument setups and measured to have $\log N\left(\mathrm{H}_{2}\right) \gtrsim 18$, indicating a self-shielded regime. We also report seven CO detections (7/39) down to $\log N(\mathrm{CO}) \sim 13.5$, including a new one, and put stringent constraints on $N(\mathrm{CO})$ for the remaining 32 systems. $N(\mathrm{CO})$ and $N(\mathrm{C} \mathrm{I})$ are found to be strongly correlated with $N(\mathrm{CO}) / N(\mathrm{C} \mathrm{I}) \sim 1 / 10$. This suggests that the $\mathrm{C}$ I-selected absorber population is probing gas deeper than the $\mathrm{H} \mathrm{I}-\mathrm{H}_{2}$ transition in which a substantial fraction of the total hydrogen in the cloud is in the form of $\mathrm{H}_{2}$. We conclude that targeting $\mathrm{C}$ I-bearing absorbers is a very efficient way to find high-metallicity molecular absorbers. However, probing the molecular content in lower-metallicity regimes as well as high-column-density neutral gas remains to be undertaken to unravel the processes of gas conversion in normal high- $z$ galaxies.
\end{abstract}

Key words. ISM: molecules - quasars: absorption lines

\section{Introduction}

The detection and analysis of molecular absorption lines along the lines of sight to background light sources has proven to be an extremely useful tool to investigate the physical and chemical state of the interstellar medium (ISM) thanks to the sensitive formation, destruction, and excitation processes of molecules. Such a technique applies from the solar neighbourhood towards nearby stars (e.g. Savage et al. 1977; Boissé et al. 2013) to the gas in and around high-redshift galaxies revealed by damped Lyman- $\alpha$ systems (DLAs; e.g. Levshakov et al. 1989; Ge et al. 1997; Petitjean et al. 2000; Cui et al. 2005; Srianand et al. 2005; Noterdaeme et al. 2008; Jorgenson et al. 2010; Carswell et al. 2011; Balashev et al. 2017). In addition, the detection of molecular species at high-redshift provides original and sensitive probes of fundamental physics and cosmology. Tiny shifts in the relative wavelengths of $\mathrm{H}_{2}$ Lyman and Werner lines can be used to constrain the possible space-time variation of the proton-to-electron mass ratio down to a few parts-per-million over a timescale of Gyrs (see Ubachs et al. 2016, and references therein). The excitation of $\mathrm{CO}$ rotational levels, in turn, provides one of the best thermometers for measuring the temperature of

\footnotetext{
* Based on observations and archival data from the European Southern Observatory (ESO) prog. IDs 060.A-9024, 072.A-0346, 278.A5062, 080.A-0482, 080.A-0795, 081.A-0242, 081.A-0334, 082.A-0544, 082.A-0569, 083.A-0454, 084.A-0699, 086.A-0074 and 086.A-0643 using the Ultraviolet and Visual Echelle Spectrograph (UVES) and X-shooter at the Very Large Telescope (VLT), on Cerro Paranal, Chile.
}

the cosmic microwave background (CMB) radiation at high redshift (Srianand et al. 2008; Noterdaeme et al. 2011). Last but not least, the molecular phase of the ISM makes the link between the gas accreted onto a galaxy and its gravitational collapse that gives birth to stars. However, the small number of known molecular absorbers contrasts with the huge number of DLAs detected so far (e.g. Prochaska et al. 2005; Noterdaeme et al. 2012): only about 25 confirmed high-redshift $\mathrm{H}_{2}$-bearing DLAs have been reported to date (see Balashev et al. 2017 and references therein), highlighting the small covering factor of the molecular gas and the need for efficient selection techniques.

In the local ISM, early works using Copernicus showed that $\mathrm{H}_{2}$ and neutral carbon ( $\mathrm{C} \mathrm{I}$ ) were frequently observed in the same absorption systems (e.g. Liszt 1981). Despite the high abundance of carbon, it is usually found in ionised forms in high-redshift DLAs and the neutral carbon is seen only in a small fraction of DLAs that also show $\mathrm{H}_{2}$ absorption (e.g. Ge et al. 2001; Srianand et al. 2005). This is likely due to the first ionisation potential of carbon $(11.26 \mathrm{eV})$ being close to the energy of Lyman-Werner photons that lead to $\mathrm{H}_{2}$ dissociation (through Solomon process, see e.g. Stecher \& Williams 1967). C I also conveniently produces absorption lines out of the Lyman- $\alpha$ forest that can be identified even at low spectral resolution. We have therefore performed the first blind survey for neutral carbon lines in quasar spectra from the Sloan Digital Sky Survey (SDSS; Ledoux et al. 2015), without any prior knowledge of the associated atomic and molecular content. The $66 \mathrm{C}$ I candidates constitute our parent sample. We report here on the complete follow up of this sample 
with the Ultraviolet and Visual Echelle Spectrograph (UVES) at a resolving power $R \sim 50000$ and the X-shooter spectrograph $(R \sim 5000)$ at the Very Large Telescope (VLT).

\section{Observations and results}

We obtained spectra for almost all systems that are observable from Paranal Observatory, that is, a sample of thirty nine confirmed C I absorbers. Details about the observing procedures, data reduction, and metal line measurements are presented in Ledoux et al. (in prep.). A near-infrared study of the Na I and $\mathrm{Ca}$ II lines as well as the dust extinction properties are presented in Zou et al. (2018). Here, we focus on the detection of $\mathrm{H}_{2}$ and $\mathrm{CO}$. Wavelengths and oscillator strengths for $\mathrm{H}_{2}$ and $\mathrm{CO}$ lines are from the compilations of Malec et al. (2010) and Daprà et al. (2016), respectively.

\subsection{Molecular hydrogen}

We detect $\mathrm{H}_{2}$ absorption lines whenever covered by our spectra (twelve systems). Five of these are already reported in the literature (Noterdaeme et al. 2007, 2010; Srianand et al. 2008; Jorgenson et al. 2010; Klimenko et al. 2016), from which we have taken the $\mathrm{H}_{2}$ column densities, and seven are new detections. We estimated the total $\mathrm{H}_{2}$ column densities for the new detections through Voigt-profile fitting, focusing on the low rotational levels that contain most of the $\mathrm{H}_{2}$. We note that while the velocity profile of singly ionised metals is wide with a large number of components, we detect $\mathrm{H}_{2}$ only at velocities where $\mathrm{C} \mathrm{I}$ is also detected. Below we comment on each system, in order of increasing right ascension of the background quasar.

$\mathrm{J} 091721+015448, z_{\mathrm{abs}}=2.107$ : this system was observed with X-shooter at a spectral resolution of $\sim 60 \mathrm{~km} \mathrm{~s}^{-1}$. We obtain an accurate measurement of the total $\mathrm{H}_{2}$ column density thanks to the damping wings that are seen for the low rotational levels in the four bands covered by our spectrum (see Fig. 1) and obtain $\log N\left(\mathrm{H}_{2}\right)=20.11 \pm 0.06$.

$\mathrm{J} 111756+143716, z_{\mathrm{abs}}=2.001:$ this system is characterised by two narrow $\mathrm{H}_{2}$ components seen in the UVES spectrum (Fig. 2) in different rotational levels. These components also correspond to those seen in the neutral carbon lines. While our best-fit value is found to be around $\log N\left(\mathrm{H}_{2}\right) \sim 18$, we note that the data quality is poor and that only one band is covered, making it impossible to assess the presence of blends. In addition, at such a column density, the absorption is in the logarithmic part of the curve of growth. We are therefore unable to associate an uncertainty to this measurement that we display with a large arbitrary (albeit quite conservative) 1 dex error bar in Fig. 9.

$\mathrm{J} 131129+222552, z_{\mathrm{abs}}=3.092$ : thanks to the high absorption redshift, no less than twenty Lyman and Werner $\mathrm{H}_{2}$ bands are covered by our UVES spectrum, shown in Fig. 3. Four components can be distinguished in the high rotational levels but lines from the $J=0$ and $J=1$ rotational levels are strongly damped and therefore modelled using a single component. The damping wings together with the large number of detected transitions and the achieved high signal-to-noise ratio $(\mathrm{S} / \mathrm{N})$ values allow for a very precise measurement of the total $\mathrm{H}_{2}$ column density which we found to be $\log N\left(\mathrm{H}_{2}\right)=19.69 \pm 0.01$.

$\mathrm{J} 164610+232922, z_{\mathrm{abs}}=1.998:$ while the $\mathrm{S} / \mathrm{N}$ of our UVES spectrum in the region of $\mathrm{H}_{2}$ lines (see Fig. 4) is quite low ${ }^{1}$, two

1 Although SDSS J164610+232922 is a relatively bright quasar $(g=$ 18.5 ), only a single $4000 \mathrm{~s}$ exposure could be obtained at a high airmass (1.7).

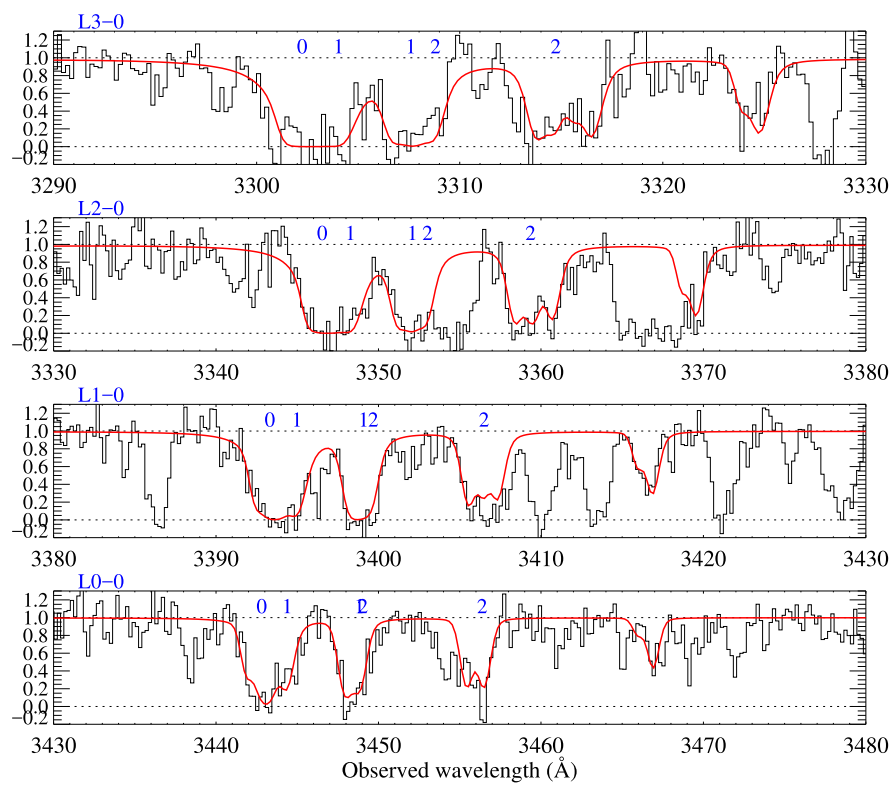

Fig. 1. Selected regions of the X-shooter spectrum of J0917+0154 featuring $\mathrm{H}_{2}$ lines. The rotational levels $J=0$ to $J=2$ are indicated in blue above their corresponding absorption line and the label above each panel indicates the band they belong to. Higher rotational levels are fitted but not labelled to avoid overcrowding the figure.

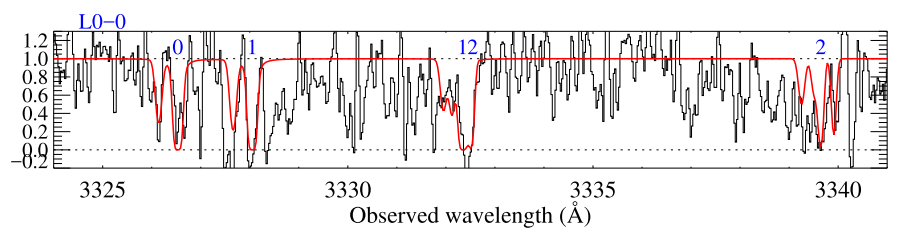

Fig. 2. As in Fig. 1 but for the UVES spectrum of J1117+1437.

narrow $\mathrm{H}_{2}$ components are clearly seen in rotational levels $J=$ 0-3 and our spectrum covers four Lyman bands that span more than an order of magnitude in oscillator strengths. We find a total column density $N\left(\mathrm{H}_{2}\right) \approx 10^{18} \mathrm{~cm}^{-2}$ with a $\sim 30 \%$ uncertainty.

$\mathrm{J} 225719-100104, z_{\mathrm{abs}}=1.836$ : this system is more complex with no less than eight $\mathrm{H}_{2}$ components, strongly blended with each other. Unfortunately, only three Lyman $\mathrm{H}_{2}$ bands are covered by the UVES data (Fig. 5), the bluest of which in a region with low $\mathrm{S} / \mathrm{N}$. To remove strong degeneracy between parameters, we had to fix the excitation temperature $T_{01}$ to $100 \mathrm{~K}$. While this is a strong assumption, we note that varying $T_{01}$ within a factor of two has little effect on the total column $\mathrm{H}_{2}$ density (changes $\sim 0.1$ dex). Still, we caution that this error may be underestimated and covering bluer transitions is required to confirm our column density measurement $\left(\log N\left(\mathrm{H}_{2}\right)=19.5 \pm 0.1\right)$.

$\mathrm{J} 233156-090802, z_{\mathrm{abs}}=2.142$ : in spite of the low $\mathrm{S} / \mathrm{N}$ achieved for this system, shown in Fig. 6, the data is clearly consistent with strongly damped $\mathrm{H}_{2}$ lines at the same redshift as that of CO lines (see following section). We fitted the $J=0,1,2$ lines, from which we obtain realistic excitation temperatures, $T_{01} \sim 140 \mathrm{~K}$ and $T_{02} \sim 180 \mathrm{~K}$. The total $\mathrm{H}_{2}$ column density is found to be $\log N\left(\mathrm{H}_{2}\right)=20.57 \pm 0.05$.

$\mathrm{J} 233633-105841, z_{\mathrm{abs}}=1.829$ : the $\mathrm{H}_{2}$ profile in this system is well modelled by two components, that are partially blended at the X-shooter spectral resolution. The bluest component, however, dominates the total column density, and the measurement is facilitated by the presence of damping wings and the high $\mathrm{S} / \mathrm{N}$ achieved. We note that the $L 0-0$ band is partially blended with 


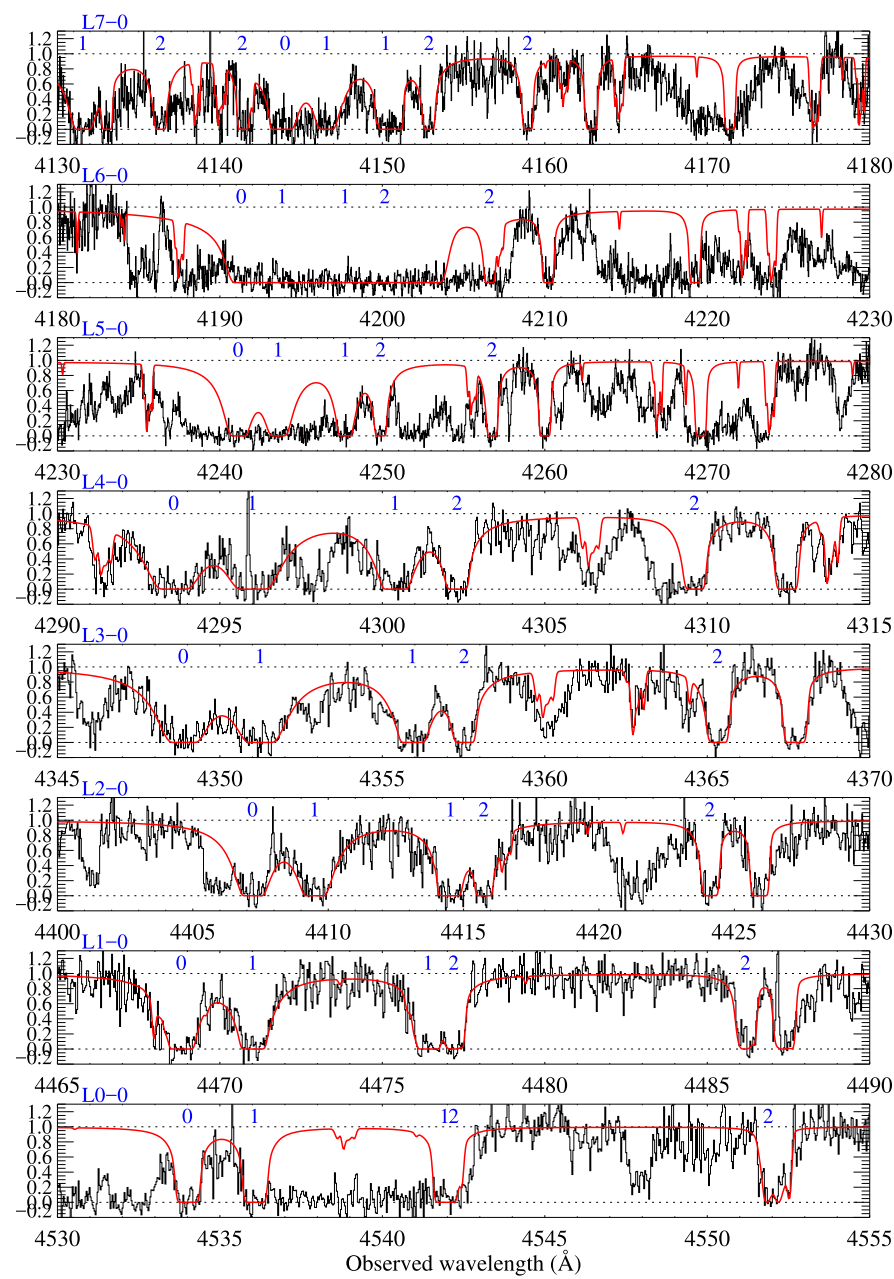

Fig. 3. As in Fig. 1 but for the UVES spectrum of J1311+2225. We note that different bands start to overlap which each other at the shortest wavelength. As for other systems, the label indicated at the top left of each panel corresponds to the band to which the identified rotational levels belong.

unrelated absorption lines, which we modelled when fitting $\mathrm{H}_{2}$ (see Fig. 7). We obtain $\log N\left(\mathrm{H}_{2}\right)=19.0 \pm 0.12$.

\subsection{Carbon monoxide}

$\mathrm{CO}$ is detected in seven systems in our sample, six of them already reported by our group and one being a new detection presented here for the first time. This brings the number of known high- $z$ CO-bearing quasar absorbers to nine ${ }^{2}$. We measured upper limits on $N(\mathrm{CO})$ for all systems assuming the Doppler parameter to be $1 \mathrm{~km} \mathrm{~s}^{-1}$, similar to what has been measured in all high- $z$ CO absorbers to date. We also assume the CMB radiation to be the main excitation source in diffuse gas at high- $z$ (as observed by Srianand et al. 2008; Noterdaeme et al. 2011).

We calculated the local (i.e. for each band individually) and global $\chi^{2}$ values for a range of total column densities. $\mathrm{CO}$ is detected when the $\chi^{2}$ curves are consistent with each other and present a clear inflexion point, defining the best-fit value. For non-detections, $\chi^{2}(\mathrm{~N}(\mathrm{CO}))$ is generally monotonic with a minimum consistent with that of $N(\mathrm{CO})=0$ within uncertainty.

2 The detections towards J1211 +0833 (Ma et al. 2015) and J0000+0015 (Noterdaeme et al. 2017) are not formally part of the statistical sample although selected upon their $\mathrm{C}$ I content.
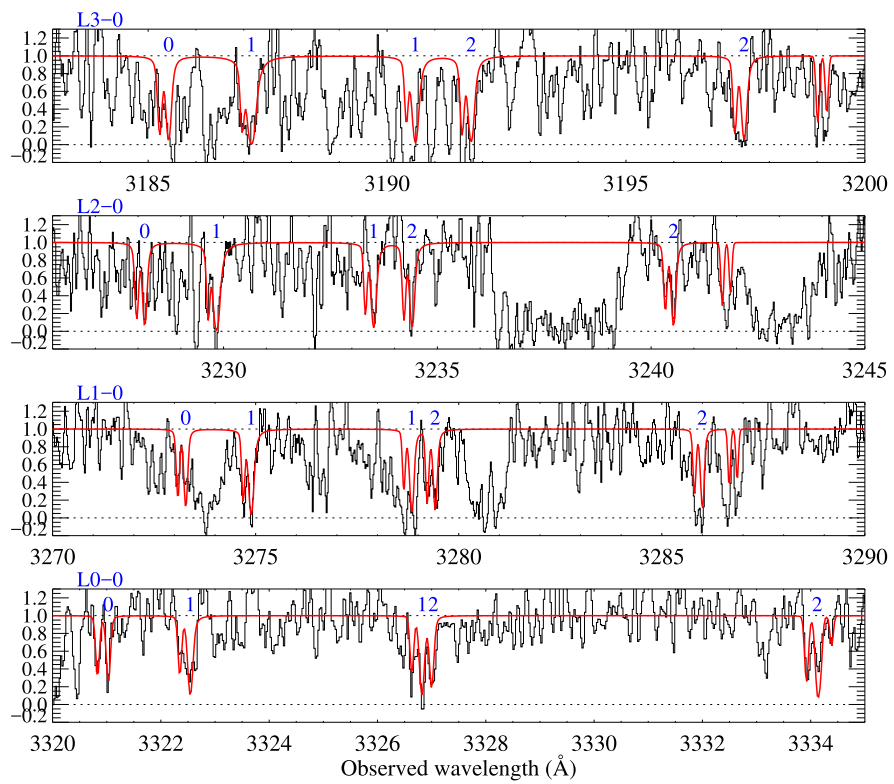

Fig. 4. As in Fig. 1 but for the UVES spectrum of J1646+2329.

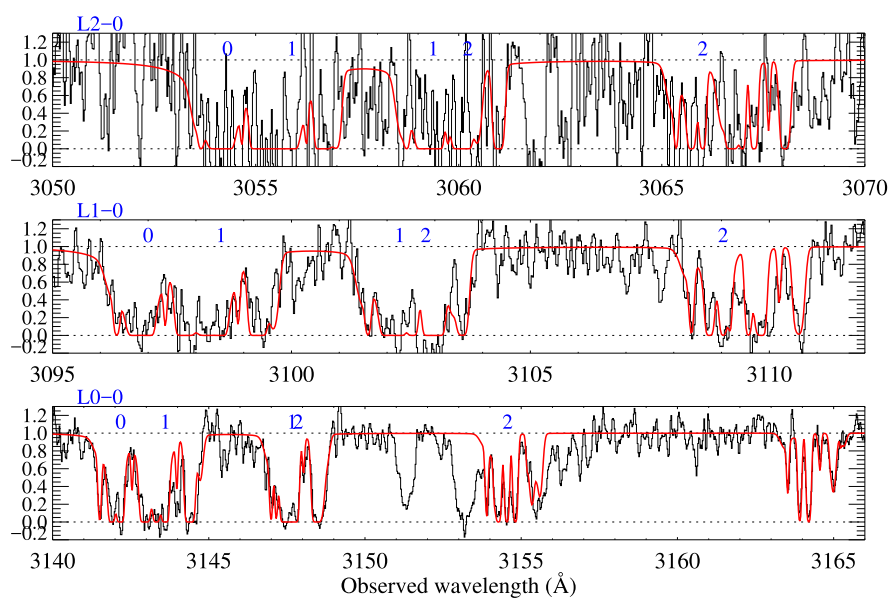

Fig. 5. As in Fig. 1 but for the UVES spectrum of J2257-1001.

Our $3 \sigma$ upper limit corresponds to the column density where the $\chi^{2}$ is 9 above this minimum. With this method, not only do we recover all the known $\mathrm{CO}$ absorbers but we also identify the new CO system, at $z=2.143$ towards SDSS J2331-0908 (Fig. 8), observed by Nestor and collaborators (Prog. ID 080.A0795). This is only the fourth high- $z$ system with direct and simultaneous measurements of $N(\mathrm{CO})$ and $N\left(\mathrm{H}_{2}\right)$.

Before discussing our findings, it is worth mentioning that, in the local ISM, the excitation temperature of $\mathrm{CO}$ is found to be a few degrees above the CMB temperature (e.g. Burgh et al. 2007), owing to additional excitation processes such as collisions, farinfrared dust emission, and possibly cosmic rays. Relaxing our assumptions we find that the derived $\mathrm{CO}$ column density limits (as well as the CO column density for the new detection at $z_{\text {abs }}=2.143$ towards SDSS J2331-0908) are not changed significantly as the total band equivalent width is almost conserved. For example, allowing an excitation temperature $5 \mathrm{~K}$ above the CMB temperature only increases the derived values by less than 0.04 dex. 


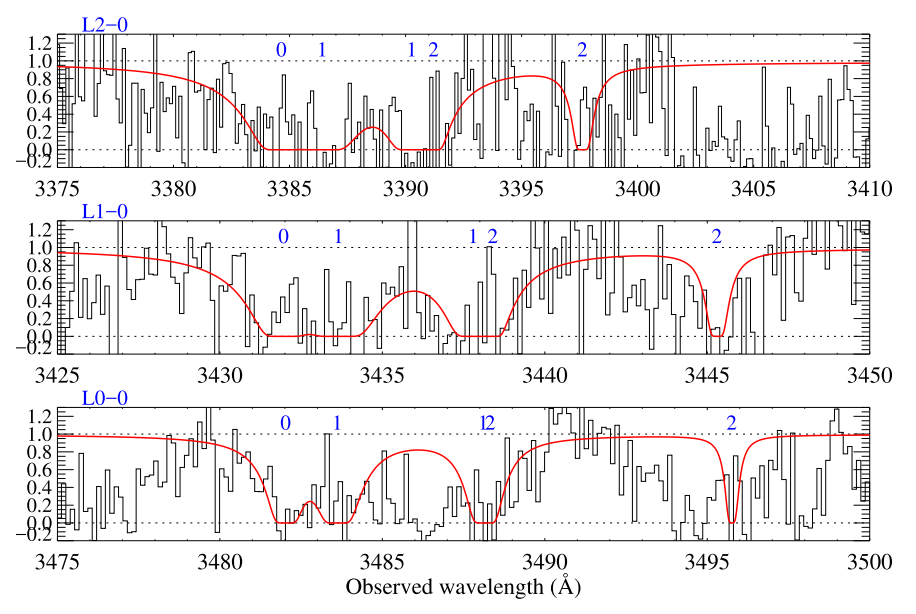

Fig. 6. As in Fig. 1 but for the UVES spectrum of J2331-0908. The data have been rebinned by 7 pixels for visual purposes only.

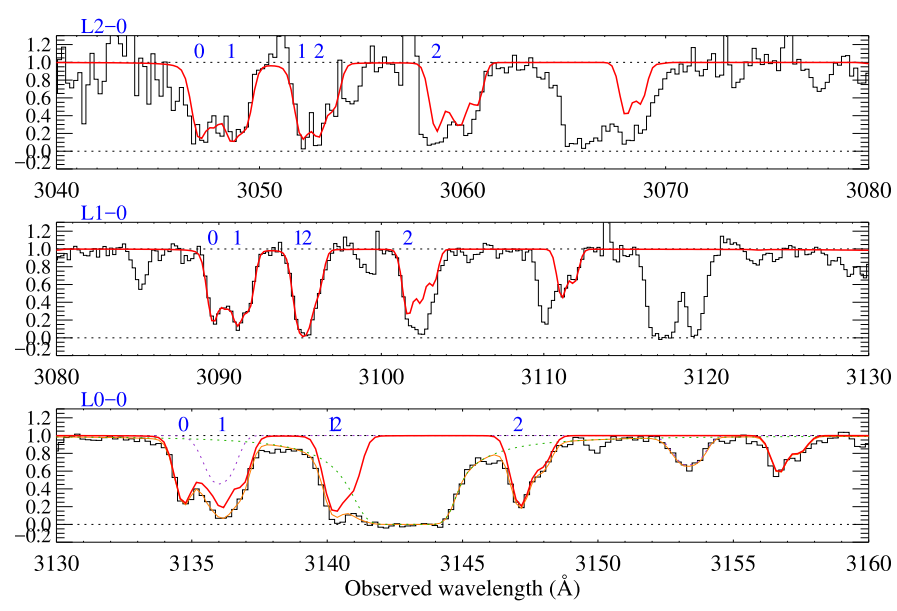

Fig. 7. As in Fig. 1 but for the X-shooter spectrum of J2336-1058. The green and purple dotted lines in the bottom panel show the contribution from unrelated $\operatorname{Ly} \alpha$ (from a sub-DLA at $z_{\mathrm{abs}}=1.585$ ) and $\mathrm{O}$ VI $\left(z_{\mathrm{abs}}=2.039\right)$ absorption, respectively. The contribution from $\mathrm{H}_{2}$ alone is shown in red and the total absorption-line profile is depicted in orange.

\section{Discussion}

Table 1 summarises the $\mathrm{H}_{2}$ and $\mathrm{CO}$ detections and column density measurements. Figure 9 presents the $\mathrm{H}_{2}$ and $\mathrm{CO}$ column densities as well as the overall molecular fraction $\left\langle f_{\mathrm{H} 2}\right\rangle=$ $2 N\left(\mathrm{H}_{2}\right) /\left(2 N\left(\mathrm{H}_{2}\right)+N(\mathrm{HI})\right)$ as a function of $N(\mathrm{C} \mathrm{I})$ for our complete sample. Known systems from the literature are also added for comparison but not considered for statistical analysis.

We find that $\mathrm{H}_{2}$ is detected with $N\left(\mathrm{H}_{2}\right) \gtrsim 10^{18} \mathrm{~cm}^{-2}$ in all systems with $\log N(\mathrm{CI})>13.5$. In this regime, $\mathrm{H}_{2}$ is likely to be self-shielded and the molecular fraction substantial in the $\mathrm{H}_{2}$-bearing gas. We also observe a possible trend for increasing $N\left(\mathrm{H}_{2}\right)$ with increasing $N(\mathrm{C} \mathrm{I})$ (Spearman rank correlation coefficient $r=0.4,1.2 \sigma$ significance) in our statistical sample, albeit with a large dispersion. We note that systems that were not $\mathrm{C}$ I-selected (from literature) seem not to follow this trend. Four of them indeed have $N\left(\mathrm{H}_{2}\right)>5 \times 10^{19} \mathrm{~cm}^{-2}$ in spite of relatively low $\mathrm{C}$ I column density $(\log N(\mathrm{C} \mathrm{I}) \lesssim 14)$. This difference is likely due to the different chemical enrichments: C I-selected systems probe mostly high-metallicity gas (Zou et al. 2018; Ledoux et al., in prep.) while the four mentioned literature systems all have low metallicities.
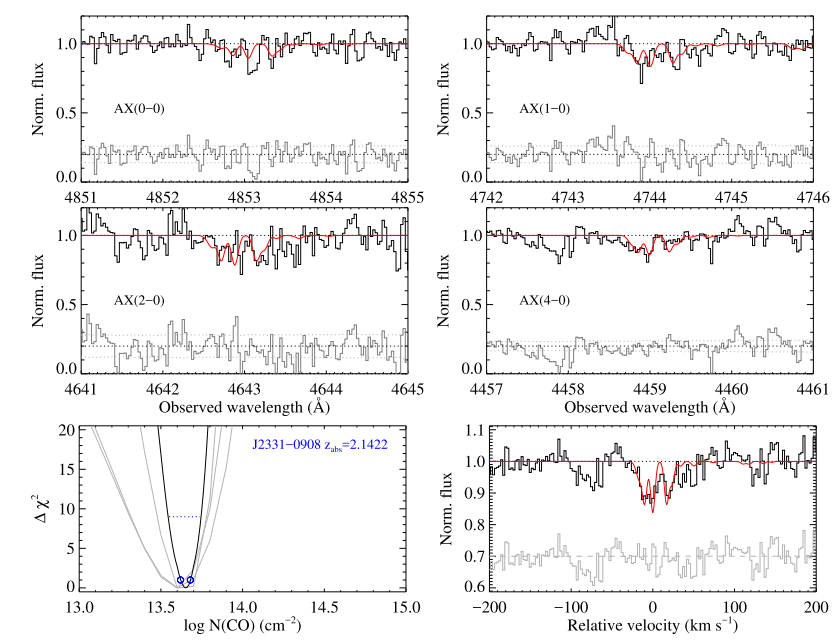

Fig. 8. $\mathrm{CO} A X$ bands at $z_{\mathrm{abs}}=2.1422$ towards J2331-0908 (top four panels, $A X(3-0)$ is not covered by the instrument setup). The bottom-right panel shows a co-addition of the CO bands, using $(f / \sigma)^{2}$ weighting, where $f$ is the oscillator strength and $\sigma$ the uncertainty on the normalised flux, for easy visualisation of the detection. The bottomleft panel shows the $\Delta \chi^{2}=\chi^{2}-\chi_{\min }^{2}$ curves (grey for individual band, black for total).

Since the column density at which $\mathrm{H} \mathrm{I}$ is converted into $\mathrm{H}_{2}$ strongly depends on the chemical properties of the gas, in particular the abundance of dust grains (e.g. Bialy \& Sternberg 2016), we can expect less H I in the molecular cloud envelope for high-metallicity systems compared to low-metallicity ones. In addition, contrary to DLAs, C I systems were selected without any prior knowledge of the H I content (Ledoux et al. 2015) and should have less contribution from unrelated atomic gas that does not belong to the envelope of the $\mathrm{H}_{2}$ cloud. This is seen in the bottom panel of Fig. 9, where the correlation between $\langle f\rangle$ and $N(\mathrm{C} \mathrm{I})$ is seen with $r=0.6$ at $2.1 \sigma$ : the average overall $\mathrm{H}_{2}$ molecular fraction is about $15 \%$ in our sample (and about $30 \%$ when $\mathrm{CO}$ is detected) but $<3 \%$ at $\log N(\mathrm{C} \mathrm{I})<14$.

The correlation between $N(\mathrm{CO})$ and $N(\mathrm{C}$ I $)$ for $\mathrm{CO}$ detections is strong with $r=0.88(2.6 \sigma)$. From the column density distributions, we can see that the probability to detect $\mathrm{CO}$ becomes much larger above $N(\mathrm{CI}) \sim 5 \times 10^{14} \mathrm{~cm}^{-2}(6 / 12)$ than below this value (1/27). In addition, there is no $\mathrm{CO}$ detection among the 18 systems with $\log N(\mathrm{CI})<14.4$. Since the $\mathrm{CO}$ detection limits are significantly below $(\sim 1 \mathrm{dex})$ the typical $N(\mathrm{CO})$ in the case of detection, this result is robust ${ }^{3}$. Considering also lower and upper limits on both $N(\mathrm{CO})$ and $N(\mathrm{C} \mathrm{I})$, we still find the $N(\mathrm{CO})-N(\mathrm{C} \mathrm{I})$ correlation to have $\sim 92 \%$ probability.

This strong correlation is likely due to the strong dependence of $\mathrm{CO}$ abundance on the metallicity (through the abundance of carbon, the abundance of dust grains as molecule-formation catalyst, and an effective shielding of UV photons). In Ledoux et al. (2015), we showed that strong C I systems produce more reddening than other classes of quasar absorbers. We further note that the dust reddening is systematically higher in CO-bearing systems than other $\mathrm{H}_{2}$ systems without $\mathrm{CO}$. The relative behaviours of $\mathrm{CO}, \mathrm{H}_{2}$, and $\mathrm{C}$ I agree qualitatively with models of ISM clouds immersed in a UV radiation field: these clouds are expected to

\footnotetext{
3 We note that $N(\mathrm{CO})$-limits also tend to be more stringent for systems with low $N(\mathrm{CI})$. This is likely due to easier detection of small $\mathrm{C}$ I equivalent widths in SDSS towards bright quasars (or that such systems extinguish less the background quasars), for which the follow-up data is also of better quality.
} 
Table 1. $\mathrm{CO}$ and $\mathrm{H}_{2}$ content of strong $\mathrm{C} \mathrm{I}$ absorbers.

\begin{tabular}{|c|c|c|c|c|c|}
\hline \multirow[t]{2}{*}{ Quasar } & \multirow[t]{2}{*}{$z_{\mathrm{abs}}$} & \multicolumn{3}{|c|}{$\log N\left(\mathrm{~cm}^{-2}\right)$} & \multirow[t]{2}{*}{ Ref. } \\
\hline & & $\mathrm{C} \mathrm{I}$ & $\mathrm{CO}$ & $\mathrm{H}_{2}$ & \\
\hline J0216-0021 & 1.737 & $14.25 \pm 0.02$ & $<12.97$ & - & 1 \\
\hline J0300-0721 & 1.536 & $>14.77$ & $\leq 13.79$ & - & 1 \\
\hline J0811+0838 & 1.906 & $13.68 \pm 0.13$ & $<13.24$ & - & 1 \\
\hline $\mathrm{J} 0815+2640$ & 1.681 & $>14.73$ & $<13.68$ & - & 1 \\
\hline $\mathrm{J} 0820+1559$ & 1.547 & $>14.71$ & $<14.01$ & - & 1 \\
\hline J0852+1935 & 1.788 & $15.01 \pm 0.12$ & $<13.33$ & - & 1 \\
\hline J0854+0317 & 1.567 & $14.23 \pm 0.01$ & $<13.11$ & - & 1 \\
\hline $\mathrm{J} 0857+1855$ & 1.730 & $14.57 \pm 0.13$ & $13.54 \pm 0.05$ & - & 2 \\
\hline J0917+0154 & 2.107 & $14.32 \pm 0.06$ & $<14.07$ & $20.11_{-0.06}^{+0.06}$ & 1 \\
\hline $\mathrm{J} 0927+1543$ & 1.731 & $>14.61$ & $<13.32$ & $--^{-0.06}$ & 1 \\
\hline $\mathrm{J} 1047+2057$ & 1.775 & $>14.90$ & $14.40 \pm 0.07$ & - & 2 \\
\hline $\mathrm{J} 1117+1437$ & 2.001 & $14.40 \pm 0.03$ & $<13.13$ & $\sim 18$ & 1 \\
\hline $\mathrm{J} 1122+1437$ & 1.554 & $13.83 \pm 0.03$ & $<12.99$ & - & 1 \\
\hline J1129-0237 & 1.623 & $>14.96$ & $<13.31$ & - & 1 \\
\hline $\mathrm{J} 1133-0057$ & 1.706 & $15.12 \pm 0.06$ & $\leq 14.07$ & - & 1 \\
\hline $\mathrm{J} 1237+0647$ & 2.691 & $15.01 \pm 0.02$ & $14.17 \pm 0.09$ & $19.21_{-0.12}^{+0.13}$ & 3 \\
\hline $\mathrm{J} 1248+2848$ & 1.513 & $14.25 \pm 0.10$ & $<13.25$ & $-^{-0.12}$ & 1 \\
\hline $\mathrm{J} 1302+2111$ & 1.656 & $14.30 \pm 0.02$ & $<13.48$ & - & 1 \\
\hline $\mathrm{J} 1306+2815$ & 2.012 & $14.47 \pm 0.04$ & $<13.26$ & - & 1 \\
\hline $\mathrm{J} 1311+2225$ & 3.092 & $14.30 \pm 0.02$ & $<13.43$ & $19.69_{-0.01}^{+0.01}$ & 1 \\
\hline $\mathrm{J} 1314+0543$ & 1.583 & $14.40 \pm 0.02$ & $\leq 13.77$ & $-^{-0.01}$ & 1 \\
\hline $\mathrm{J} 1341+1852$ & 1.544 & $13.51 \pm 0.03$ & $<13.00$ & - & 1 \\
\hline $\mathrm{J} 1346+0644$ & 1.512 & $14.51 \pm 0.02$ & $<13.60$ & - & 1 \\
\hline $\mathrm{J} 1439+1117$ & 2.418 & $14.81 \pm 0.02$ & $13.89 \pm 0.02$ & $19.38_{-0.10}^{+0.10}$ & 4 \\
\hline $\mathrm{J} 1459+0129$ & 1.623 & $14.32 \pm 0.09$ & $<13.57$ & - & 1 \\
\hline $\mathrm{J} 1522+0830$ & 1.627 & $>14.47$ & $<13.50$ & - & 1 \\
\hline $\mathrm{J} 1603+1701$ & 1.890 & $13.80 \pm 0.10$ & $<12.88$ & - & 1 \\
\hline $\mathrm{J} 1604+2203$ & 1.641 & $>15.14$ & $14.59 \pm 0.11$ & - & 5 \\
\hline $\mathrm{J} 1615+2648$ & 2.118 & $14.49 \pm 0.06$ & $<13.16$ & - & 1 \\
\hline $\mathrm{J} 1623+1355$ & 1.751 & $14.41 \pm 0.07$ & $<13.30$ & - & 1 \\
\hline $\mathrm{J} 1646+2329$ & 1.998 & $14.32 \pm 0.06$ & $<13.40$ & $18.02_{-0.11}^{+0.11}$ & 1 \\
\hline $\mathrm{J} 1705+3543$ & 2.038 & $>15.01$ & $14.14 \pm 0.03$ & - & 2 \\
\hline $\mathrm{J} 2123-0050$ & 2.060 & $14.11 \pm 0.02$ & $<13.07$ & $17.94_{-0.01}^{+0.01}$ & 1,6 \\
\hline $\mathrm{J} 2229+1414$ & 1.586 & $13.96 \pm 0.05$ & $<13.55$ & - & 1 \\
\hline $\mathrm{J} 2257-1001$ & 1.836 & $14.65 \pm 0.01$ & $<13.09$ & $19.5 \pm 0.1$ & 1 \\
\hline J2331-0908 & 2.143 & $>14.70$ & $13.65 \pm 0.03$ & $20.57_{-0.05}^{+0.05}$ & 1 \\
\hline $\mathrm{J} 2336-1058$ & 1.829 & $14.07 \pm 0.02$ & $<12.93$ & $19.00_{-0.12}^{+0.12}$ & 1 \\
\hline $\mathrm{J} 2340-0053$ & 2.054 & $14.09 \pm 0.04$ & $<12.58$ & $18.47_{-0.04}^{+0.04}$ & 1,7 \\
\hline $\mathrm{J} 2350-0052$ & 2.426 & $14.36 \pm 0.01$ & $<12.94$ & $18.52_{-0.49}^{+0.29}$ & 1,8 \\
\hline
\end{tabular}

References. The references listed in the last column are for molecular measurements. When two references are listed, they correspond to $\mathrm{CO}$ then $\mathrm{H}_{2}$, in this order. (1) This work; (2) Noterdaeme et al. (2011); (3) Noterdaeme et al. (2010); (4) Srianand et al. (2008); (5) Noterdaeme et al. (2009); (6) Klimenko et al. (2016); (7) Jorgenson et al. (2010); (8) Noterdaeme et al. (2007). Unless already available from the literature, $\mathrm{C}$ I column densities were obtained through the apparent optical depth method.

exhibit an onion-like structure where hydrogen converts from atomic to molecular form when going towards the centre of the cloud. Carbon is predominantly ionised in the external layers, then becomes neutral, while CO is dominant only in the inner dense molecular parts of the cloud (see e.g. Bolatto et al. 2013). Unfortunately, it remains difficult to disentangle the atomic gas that belongs to a molecular cloud envelope and contributes to its shielding from unrelated $\mathrm{HI}$, simply intercepted along the same

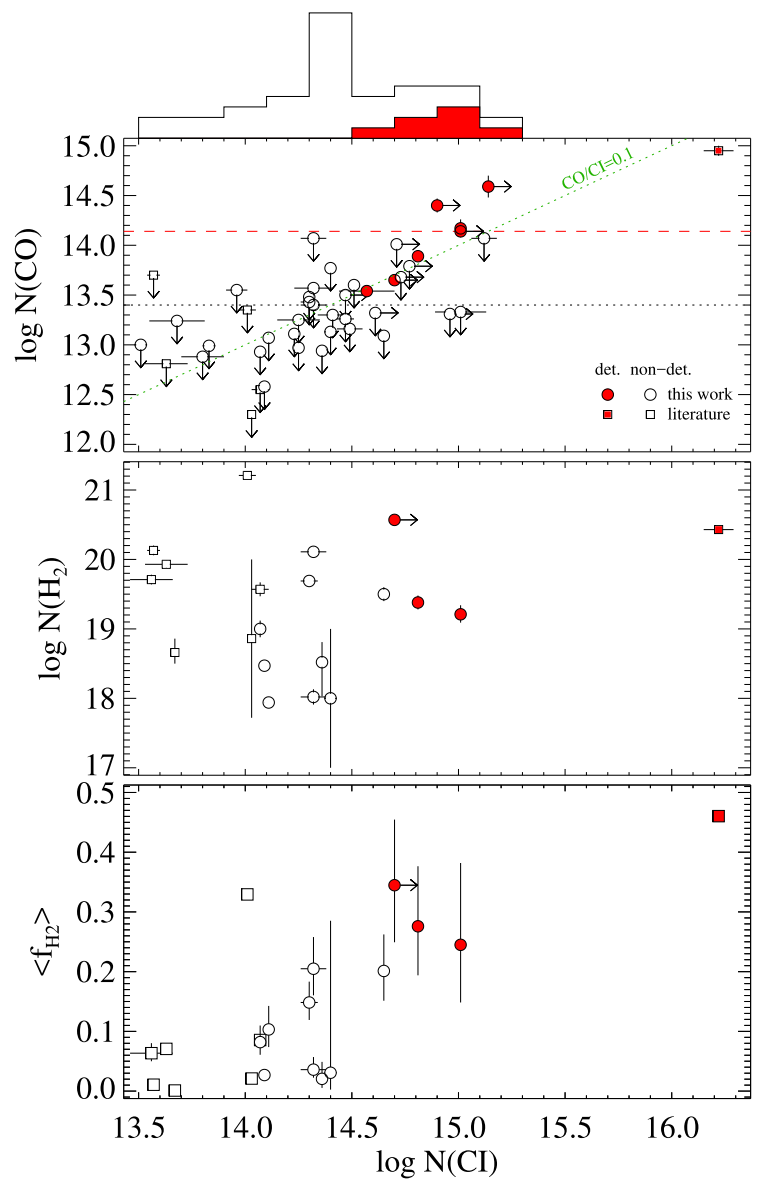

Fig. 9. Column densities of $\mathrm{CO}$ (top), $\mathrm{H}_{2}$ (middle) and overall molecular fractions (bottom) vs $N(\mathrm{C} \mathrm{I})$. CO detections are represented by red colours. The $N(\mathrm{C} \mathrm{I})$-distributions and median $\log N(\mathrm{CO})$ values (horizontal lines) are shown for the statistical sample only (circles). Squares correspond to high- $z \mathrm{H}_{2}$ DLA systems from the literature (Balashev et al. 2010, 2011, 2017; Carswell et al. 2011; Guimarães et al. 2012; Noterdaeme et al. 2015, 2017; Petitjean et al. 2002).

line of sight. This means that the measured $\langle f\rangle$ is a lower limit to the actual $\mathrm{H}_{2}$ molecular fraction in the $\mathrm{C}$ I-bearing cloud. Since $\mathrm{CO}$ and $\mathrm{C}$ I are only found in shielded gas, their observed abundance ratio should be less affected by the presence of unrelated gas. Indeed, we find $\mathrm{CO} / \mathrm{C} \mathrm{I} \sim 0.1$ for all detections (green dotted line in Fig. 9), a value which is also consistent with the nondetections at lower $N(\mathrm{C} \mathrm{I})$. This indicates a regime deeper than the layer where the $\mathrm{H} \mathrm{I}-\mathrm{H}_{2}$ transition occurs.

The $\mathrm{CO} / \mathrm{H}_{2}$ ratio is found to be low $\left(\sim[3-9] \times 10^{-6}\right)$ for three out of four cases where both these molecules are detected and can be more than an order of magnitude lower in other strong $\mathrm{H}_{2}$ systems, including the new $\mathrm{CO}$ detection. Even in these cases, the high $N\left(\mathrm{H}_{2}\right)$ likely indicates well-molecularized regions. Several factors such as the grain size distribution or the intensity of the cosmic-ray field likely play important roles in determining whether $\mathrm{CO}$ will be present or not in $\mathrm{H}_{2}$-dominated regions (e.g. Shaw et al. 2016; Noterdaeme et al. 2017; Bisbas et al. 2017). Multiple clouds can also easily explain large $\mathrm{H}_{2}$ column densities without significant $\mathrm{CO}$, in a similar way to how multiple $\mathrm{H} \mathrm{I}-\mathrm{H}_{2}$ transition layers explain higher $N(\mathrm{HI})$ than predicted by single cloud models (Bialy et al. 2017).

We conclude that $\mathrm{C} I$ is a very good proxy to spot highredshift molecular absorbers that can be used for a variety of studies including fundamental physics and cosmology. It is 
however crucial not only to constrain the physical parameters in individual systems (and hopefully for individual velocity components separately) but also to explore different metallicity regimes (Balashev et al. 2017) using different selections (e.g. Balashev et al. 2014) to understand better the molecular structure of ISM clouds at high redshifts.

Acknowledgements. We thank T. Krühler for help with the X-shooter data reduction. P.N. thanks the European Southern Observatory for hospitality and support during part of this work was done. P.N., P.P.J. and R.S. acknowledge support from the Indo-French Centre for the Promotion of Advanced Research (Project 5504-B). We acknowledge support from the PNCG funded by CNRS/INSUIN2P3-INP, CEA and CNES, France. This research is part of the project $H I H 2$ funded by the Agence Nationale de la Recherche, under grant ANR-17-CE310011-01 (JCJC). S.B. thanks the Institut d'Astrophysique de Paris for hospitality and the Institut Lagrange de Paris for financial support. S.L. has been supported by FONDECYT grant 1140838 and by PFB-06 CATA.

\section{References}

Balashev, S. A., Ivanchik, A. V., \& Varshalovich, D. A. 2010, Astron. Lett., 36, 761

Balashev, S. A., Petitjean, P., Ivanchik, A. V., et al. 2011, MNRAS, 418, 357

Balashev, S. A., Klimenko, V. V., Ivanchik, A. V., et al. 2014, MNRAS, 440, 225

Balashev, S. A., Noterdaeme, P., Rahmani, H., et al. 2017, MNRAS, 470, 2890

Bialy, S., \& Sternberg, A. 2016, ApJ, 822, 83

Bialy, S., Bihr, S., Beuther, H., Henning, T., \& Sternberg, A. 2017, ApJ, 835, 126

Bisbas, T. G., van Dishoeck, E. F., Papadopoulos, P. P., et al. 2017, ApJ, 839, 90

Boissé, P., Federman, S. R., Pineau des Forêts, G., \& Ritchey, A. M. 2013, A\&A, 559, A131

Bolatto, A. D., Wolfire, M., \& Leroy, A. K. 2013, ARA\&A, 51, 207

Burgh, E. B., France, K., \& McCandliss, S. R. 2007, ApJ, 658, 446

Carswell, R. F., Jorgenson, R. A., Wolfe, A. M., \& Murphy, M. T. 2011, MNRAS, 411,2319

Cui, J., Bechtold, J., Ge, J., \& Meyer, D. M. 2005, ApJ, 633, 649
Daprà, M., Niu, M. L., Salumbides, E. J., Murphy, M. T., \& Ubachs, W. 2016, ApJ, 826, 192

Ge, J., Bechtold, J., \& Black, J. H. 1997, ApJ, 474, 67

Ge, J., Bechtold, J., \& Kulkarni, V. P. 2001, ApJ, 547, L1

Guimarães, R., Noterdaeme, P., Petitjean, P., et al. 2012, AJ, 143, 147

Jorgenson, R. A., Wolfe, A. M., \& Prochaska, J. X. 2010, ApJ, 722, 460

Klimenko, V. V., Balashev, S. A., Ivanchik, A. V., \& Varshalovich, D. A. 2016 Astron. Lett., 42, 137

Ledoux, C., Noterdaeme, P., Petitjean, P., \& Srianand, R. 2015, A\&A, 580, A8 Levshakov, S. A., Foltz, C. B., Chaffee, F. H., \& Black, J. H. 1989, AJ, 98, 2052

Liszt, H. S. 1981, ApJ, 246, L147

Ma, J., Caucal, P., Noterdaeme, P., et al. 2015, MNRAS, 454, 1751

Malec, A. L., Buning, R., Murphy, M. T., et al. 2010, MNRAS, 403, 1541

Noterdaeme, P., Petitjean, P., Srianand, R., Ledoux, C., \& Le Petit, F. 2007, A\&A, 469,425

Noterdaeme, P., Petitjean, P., Ledoux, C., Srianand, R., \& Ivanchik, A. 2008, A\&A, 491, 397

Noterdaeme, P., Petitjean, P., Ledoux, C., \& Srianand, R. 2009, A\&A, 505, 1087

Noterdaeme, P., Petitjean, P., Ledoux, C., et al. 2010, A\&A, 523, A80

Noterdaeme, P., Petitjean, P., Srianand, R., Ledoux, C., \& López, S. 2011, A\&A, 526, L7

Noterdaeme, P., López, S., Dumont, V., et al. 2012, A\&A, 542, L33

Noterdaeme, P., Srianand, R., Rahmani, H., et al. 2015, A\&A, 577, A24

Noterdaeme, P., Krogager, J.-K., Balashev, S., et al. 2017, A\&A, 597, A82

Petitjean, P., Srianand, R., \& Ledoux, C. 2000, A\&A, 364, L26

Petitjean, P., Srianand, R., \& Ledoux, C. 2002, MNRAS, 332, 383

Prochaska, J. X., Tripp, T. M., \& Howk, J. C. 2005, ApJ, 620, L39

Savage, B. D., Bohlin, R. C., Drake, J. F., \& Budich, W. 1977, ApJ, 216, 291

Shaw, G., Rawlins, K., \& Srianand, R. 2016, MNRAS, 459, 3234

Srianand, R., Petitjean, P., Ledoux, C., Ferland, G., \& Shaw, G. 2005, MNRAS, 362,549

Srianand, R., Noterdaeme, P., Ledoux, C., \& Petitjean, P. 2008, A\&A, 482, L39

Stecher, T. P., \& Williams, D. A. 1967, ApJ, 149, L29

Ubachs, W., Bagdonaite, J., Salumbides, E. J., Murphy, M. T., \& Kaper, L. 2016, Rev. Mod. Phys., 88, 021003

Zou, S., Petitjean, P., Noterdaeme, P., et al. 2018, A\&A, in press, DOI:10.1051/0004-6361/201732033 